\title{
FNDC5 overexpression promotes the survival rate of bone marrow mesenchymal stem cells after transplantation in a rat cerebral infarction model
}

\author{
Huan Wei ${ }^{1,2}$, Kangmei Liu ${ }^{1}$, Tingting Wang ${ }^{3}$, Yanping $\mathrm{Li}^{2}$, Shaolei Guo ${ }^{4}$, Ling $\mathrm{Li}^{1}$ \\ ${ }^{1}$ Department of Neurology, The First Affiliated Hospital, Sun Yat-sen University, Guangdong Provincial Key Laboratory of Diagnosis and \\ Treatment of Major Neurological Diseases, National Key Clinical Department and Key Discipline of Neurology, Guangzhou, China; ${ }^{2}$ Department \\ of Neurology, Yan'an Hospital of Kunming City, The Affiliated Yan'an Hospital of Kunming Medical University, Kunming, China; ${ }^{3}$ Department \\ of Geriatrics, Yan'an Hospital of Kunming City, The Affiliated Yan'an Hospital of Kunming Medical University, Kunming, China; ${ }^{4}$ Department of \\ Neurosurgery, The First Affiliated Hospital, Sun Yat-sen University, Guangzhou, China \\ Contributions: (I) Conception and design: H Wei, L Li, S Guo; (II) Administrative support: None; (III) Provision of study materials or patients: None; \\ (IV) Collection and assembly of data: T Wang, Y Li; (V) Data analysis and interpretation: H Wei, K Liu; (VI) Manuscript writing: All authors; (VII) \\ Final approval of manuscript: All authors. \\ Correspondence to: Prof. Ling Li. Department of Neurology, The First Affiliated Hospital, Sun Yat-sen University, Guangdong Provincial Key \\ Laboratory of Diagnosis and Treatment of Major Neurological Diseases, National Key Clinical Department and Key Discipline of Neurology, No. \\ 58 Zhongshan Road 2, Guangzhou 510080, China. Email: liling3@mail.sysu.edu.cn; Prof. Shaolei Guo. Department of Neurosurgery, The First \\ Affiliated Hospital, Sun Yat-sen University, No. 58 Zhongshan Road 2, Guangzhou 510080, China. Email: guoshaol@mail.sysu.edu.cn.
}

Background: Most bone marrow mesenchymal stem cell (BMSC) death is caused by the harsh ischemia and hypoxic microenvironment, which impacts the therapeutic effects of transplanted BMSCs. Fibronectin type III domain-containing protein 5 (FNDC5) and its cleaved product, irisin, are reportedly involved in cerebral protective effect. Research into whether FNDC5 plays a key role in the survival rate of BMSCs and cerebral infarction (CI) remains inadequate. The present study aimed to clarify the protective role of FNDC5 on the low viability of transplanted BMSCs and improve CI treatment outcomes.

Methods: A lentivirus vector, which drives the expression of FNDC5, was constructed and used to transfect BMSCs. Cell Counting Kit-8 (CCK8), flow cytometry, immunofluorescence, and western blot were performed to evaluate the function of FNDC5-overexpressing BMSCs (BMSCs-OE-FNDC5) exposed to hypoxic and serum deprivation (H/SD) stress. Transmission electron microscopy (TEM) was used to monitor autophagy. In addition, BMSCs were engrafted into a middle cerebral artery occlusion (MCAO) rat model with or without FNDC5-overexpression (OE-FNDC5). The survival rate of transplanted BMSCs was evaluated by 5-ethynyl-2'-deoxyuridine (EdU) labeling. The CI volume was assessed by 2,3,5-triphenyl tetrazolium chloride (TTC) staining.

Results: H/SD stress caused increased cell autophagy, apoptosis, and decreased cell viability of BMSCs, while OE-FNDC5 alleviated these injuries. The in vivo results showed that transplantation of BMSCs-OEFNDC5 reduced the infarct volume in the rat MCAO model. Furthermore, OE-FNDC5 decreased neuronal apoptosis. The improved therapeutic efficacy of BMSCs-OE-FNDC5 may be attributable to the obviously increased cell survival number after transplantation.

Conclusions: These results indicated that FNDC5 overexpression promotes BMSC survival in a CI model, which might provide a potential therapeutic target.

Keywords: Fibronectin type III domain-containing protein 5 (FNDC5); bone marrow mesenchymal stem cells (BMSCs); cerebral infarction (CI); autophagy; cell viability

Submitted Nov 24, 2021. Accepted for publication Jan 11, 2022.

doi: $10.21037 /$ atm-21-6868

View this article at: https://dx.doi.org/10.21037/atm-21-6868 


\section{Introduction}

The most effective treatment of cerebral infarction (CI) should focus on the regeneration of lost neurons and the repair of neural function (1). In recent years, regenerative medicine based on mesenchymal stem cells (MSCs) has emerged as a promising approach for treatment of ischemic brain injury $(2,3)$. Previous research has shown that MSCs exert their therapeutic effect, including immune regulation, vascular regeneration, and nutritional support, through paracrine, exosomes, and differentiation mechanisms (4-6). It was originally thought that the protective efficacy of MSCs was positively correlated with the number of successfully transplanted cells. Unfortunately, the effective cell survival of grafted MSCs in infarcted brains remains unsatisfactory, with more than $80 \%$ of the infused MSCs dying within 72 hours (7). Previous studies have shown that the low survival rate of transplanted MSCs is mostly due to apoptosis or autophagy caused by the ischemic microenvironment (8), which greatly limits the therapeutic efficacy of MSCs in CI.

Fibronectin type III domain-containing protein 5 (FNDC5), a membrane protein, and its extracellular part (processed and secreted as a myokine irisin), not only play a vital role in energy metabolism but also have crucial roles in a variety of physiological processes, including inflammation, oxidative stress, proliferation, and neural differentiation. Growing evidence has demonstrated the potential role of FNDC5 in vascular activity and atherosclerosis (9-11). Recent research about stem cells has revealed that the promotion of FNDC5/Irisin can modulate osteogenic differentiation in bone marrow-derived mesenchymal stem cells (BMSCs) (12), and promote proliferation and differentiation of goat adipose-derived stem cells (13). Besides the enhancement effect on stem cells proliferation, FNDC5 has been shown to improve BMSCs engraftment and paracrine effects in infarcted hearts (14), but the underlying mechanisms remain elusive.

Growing evidence suggests that autophagy is a doubleedged sword in MSCs survival (15). In the severe ischemic microenvironment, the endoplasmic reticulum (ER) stress is uncontrollable, and the destructive autophagy of MSCs affects the regeneration ability, resulting in a string of hypoxic cell damage. Regulatory effect of FNDC5/irisin on autophagy has been studied only recently (16-18), particularly after scientists have confirmed the relationship between exerciseinduced FNDC5/irisin release and Alzheimer's disease (19).
According to the specific cellular environment, the process of FNDC5 modulating autophagy in MSCs is strictly controlled by different molecule mechanism and variable signal receptors, which needs further interpretation (20).

In this study, we conducted a series of cell and animal experiments to demonstrate whether FNDC5overexpression (OE-FNDC5) improves the therapeutic efficacy of transplanted BMSCs in ischemic brain injury, and whether the neuroprotective effects were induced, at least in part, by the inhibition of autophagy. We present the following article in accordance with the ARRIVE reporting checklist (available at https://atm.amegroups.com/article/ view/10.21037/atm-21-6868/rc).

\section{Methods}

\section{Animals and CI models}

Adult, 6-8-month-old male Sprague-Dawley (SD) rats (body weight: 280-300 g) supplied by the Guangdong Medical Laboratory Animal Center were used to establish a CI model. Animal experiments were performed under a project license (No. 2021035) granted by the Animal Care and Use Committee of The Affiliated Yan'an Hospital of Kunming Medical University, in compliance with the institutional guidelines for the care and use of animals.

Permanent middle cerebral artery occlusion (MCAO) surgery was induced by intraluminal vascular occlusion using a monofilament as previously described (21). Briefly, after the rats were anesthetized with $3 \%$ pentobarbital sodium (30 mg/kg, intraperitoneally), a midline incision was made on the neck under an operating microscope and the left common carotid artery (CCA), external carotid artery (ECA), and internal carotid artery (ICA) were isolated. A 1\% poly1-lysine-coated blunted top 6-0 suture was gently inserted $10 \pm 0.5 \mathrm{~mm}$ from the ECA into ICA to occlude the origin of the MCA. Twenty-four hours after the MCAO model was successfully prepared, Dulbecco's Modified Eagle Medium (DMEM) solution with/without BMSCs $\left(1 \times 10^{6}\right.$ cells $/ 3 \mu \mathrm{L}$ DMEM solution) was injected around the infarct border zone in a stereotactic manner. After 7 days, subsequent experiments were carried out. Rats (34 in total, six rats died either during or after the surgery) were randomly divided into three groups: MCAO + BMSCs group (BMSCs, $\mathrm{n}=10), \mathrm{MCAO}+\mathrm{BMSC}$-overexpression-negative control group (BMSCs-OE-NC, n=9), and MCAO + FNDC5overexpression BMSCs group (BMSCs-OE-FNDC5, n=9). 


\section{Lentivirus vector construction and BMSCs transfection}

The BMSCs were purchased from Cyagen Biosciences Co., Guangzhou, and cultured as previously described (22). BMSCs-OE-FNDC5 were established by transfection with lentiviruses, and a standard protocol was used to generate viruses. Briefly, lentiviruses were prepared using the $\mathrm{VsVg}$ and PAX2 lentiviral package system (Invitrogen, Carlsbad, CA, USA) using Lipofectamine 2000 (Invitrogen, Carlsbad, CA, USA) in HEK293FT cells. Supernatants containing viral particles were collected 3 days after transfection and concentrated using centrifugal cutoff filters (Merck, Darmstadt, Germany).

After dissociation into single-cell suspensions, the BMSCs were replaced with concentrated lentiviral particles. Twenty-four hours after infection, a fresh culture medium was slowly replaced. Puromysin (Sigma-Aldrich, St. Louis, MO, USA) at a concentration of $1-5 \mu \mathrm{g} / \mathrm{mL}$ was added to the culture medium 4 days after transduction. Five days later, the conventional culture medium without puromysin was replaced. Ultimately, BMSCs-OE-FNDC5 and BMSCs-OE-NC were obtained.

\section{Hypoxia/serum deprivation injury}

The BMSCs were cultured in DMEM solution containing $10 \%$ fetal bovine serum (FBS). To mimic ischemia injury in vitro, the BMSCs were exposed to a hypoxia/serum deprivation (H/SD) environment, in which cells were incubated under an atmosphere of $1 \%$ oxygen, $5 \%$ carbon dioxide, and 94\% nitrogen, in glucose-free DMEM without FBS at $37{ }^{\circ} \mathrm{C}$ for $24 \mathrm{~h}$. For normal culture, cells were conventionally incubated without H/SD.

\section{Measurement of BMSCs autophagy, apoptosis and cell viability}

The number and morphology of autophagosomes in BMSCs were observed by transmission electron microscopy (TEM, H-7650 Hitachi Limited, Japan). Apoptosis assays were performed with Annexin V-FITC Apoptosis detection kit (BD Biosciences, USA) following the manufacturer's instructions and analyzed by a FACS Aria II flow cytometer (BD Biosciences, USA). The proliferation of the cells was measured using the Cell Counting Kit-8 (CCK8; Dojindo Molecular Technologies, Japan) according to the manufacturer's protocols. The autophagy representative protein, LC3, was detected by immunofluorescence.
Autophagy biomarkers, Beclin-1 and p62, and apoptotic proteins, Bcl-2 and Bax, were detected by western blotting.

\section{Evaluation of BMSCs engraftment and histological analysis}

The survival and proliferation rates of transplanted BMSCs around the infarct zone were detected by labeling cells with EdU.

The survival of BMSCs was detected by identification of red fluorescent protein (RFP) under fluorescence microscopy, and the percentage of RFP+/4,6-diamidino-2phenylindole (DAPI) in each slide from five frozen section was calculated. All assays were performed in a blinded manner.

Terminal-deoxynucleoitidyl Transferase Mediated Nick End Labeling (TUNEL) staining was performed in brain sections using the TUNEL assay kit (Merck, Darmstadt, Germany). According to the manufacturer's protocol. Quantification of apoptosis staining was expressed as (TUNEL-stained nuclei/DAPI-stained nuclei) $\times 100 \%$. Fluorescence images were captured using a Zeiss fluorescence microscope (Leica, Barnack, Germany).

\section{Cerebral infarct volume measurement}

The measurement of cerebral infarct volume was carried out 7 days after BMSCs transplantation using 2,3,5-triphenyl tetrazolium chloride (TTC) staining. The fresh brain slices were incubated in $2 \%$ TTC at $37^{\circ} \mathrm{C}$ for $20 \mathrm{~min}$. The coronal infarction area was determined by measuring the white area of the brain section with image analysis software (National Institutes of Health, Bethesda, MD, USA) as described previously (23).

\section{Western blot analysis}

The collected cell or tissue samples were lysed for protein extraction, and the protein concentrations were measured using the Bradford assay (Thermo, Hercules, CA, USA). Protein samples were subjected to standard sodium dodecyl sulfate polyacrylamide gel electrophoresis (SDS-PAGE) and transferred to polyvinylidene fluoride membranes. After blocking in 5\% non-fat milk for $1 \mathrm{~h}$, the membranes were incubated with primary antibody against Bcl-2, Bax, Beclin1, p62, FNDC5 (Cell Signaling Technology, Boston, USA), and glyceraldehyde-phosphate dehydrogenase (GAPDH) (Santa Cruz, CA, USA) at $4{ }^{\circ} \mathrm{C}$ for $12 \mathrm{~h}$. After 
washing with buffer, the membranes were incubated with the corresponding horseradish peroxidase-conjugated secondary antibodies (Abclonal, Wuhan, China) for $1 \mathrm{~h}$. Protein bands were visualized using the BioRad imaging system (Bio-Rad, CA, USA).

\section{Quantitative real-time polymerase chain reaction (qRT-PCR)}

Total ribonucleic acid (RNA) from cells was isolated using Trizol reagent (Invitrogen, Carlsbad, CA, USA) according to the manufacturer's instructions. RNA preparations were treated using PrimeScript Reverse Transcriptase (Seyotin, Guangzhou, China) to generate complementary deoxyribonucleic acids (cDNAs). Real-time polymerase chain reaction (RT-PCR) was performed using DNA Master SYBR Green I mix (Seyotin, Guangzhou, China) on a QuantStudio 6 Flex Realtime PCR system (Applied Biosystems, Carlsbad, CA, USA). FNDC5 primer sequences were as follows: 5'-TAA CCG TCA GGC ACC TCA AG3' (forward) and 5'-CGC AGC ATC CTC ACA TCC TT3' (reverse).

\section{Statistics analysis}

All experiments were repeated more than three times. Comparisons between multiple groups was analyzed using analysis of variance (ANOVA) followed by the Tukey post hoc test. The Student's $t$ test (unpaired, two-tailed) was used to analyze the statistical differences between two groups. $\mathrm{P}$ value $<0.05$ was considered statistically significant $\left.{ }^{*}, \mathrm{P}<0.05 ;{ }^{* *}, \mathrm{P}<0.01\right)$. All quantitative data were presented as the mean \pm standard deviation (SD). SPSS (version 21.0, IBM, USA) was used to analyze the data.

\section{Results}

H/SD exposure destroyed cell viability, increased apoptosis and autophagy, and reduced the expression of FNDC5 in BMSCs

To analyze BMSC cell viability and apoptosis under H/SD stress, CCK8 assays and flow cytometry were performed as previous described. The results suggested that compared with normal conditions, the viability of BMSCs was significantly reduced $24 \mathrm{~h}$ after exposure to H/ $\mathrm{SD}$ conditions (Figure 1A). Correspondingly, the hypoxia stress increased the apoptosis rate of BMSCs (Figure 1B). In addition, immunofluorescence analysis showed that the level of LC3 in BMSCs was significantly increased (Figure 1C), which highlighted the increasing destructive autophagy of BMSCs under H/SD stress. Moreover, both qRT-PCR (Figure 1D) and western blotting (Figure 1E) results showed the decreased expression of FNDC5 in BMSCs $24 \mathrm{~h}$ after hypoxia injury.

\section{Identification and efficiency of gene transduction}

The discovery of decreased expression of FNDC5 in a hypoxic environment inspired us to investigate whether OE-FNDC5 could exert a protective effect on BMSCs. The lentivirus vector construction and transfection were described in the Methods. The BMSCs-OE-FNDC5 were obtained after antibiotic selection, and immunofluorescence staining analysis showed that BMSCs infected with OEFNDC5 or a control lentiviral vector (OE-NC) had stable viability in in vitro cultures (Figure $2 A$ ). The qRT-PCR (Figure 2B) and western blot (Figure 2C) results further confirmed the successful overexpression of FNDC5 in BMSCs.

\section{FNDC5 overexpression promoted cell proliferation and ameliorated apoptosis}

To evaluate the protective effects of OE-FNDC5 on BMSCs viability in vitro, EdU-Green Fluorescent Proteins (GFP) immunofluorescence staining was performed. The results illustrated the protective role of FNDC5 in reversing the H/SD stress-induced impaired cell proliferation (Figure $3 A$ ). The flow cytometry results further confirmed that increased cellular apoptosis was significantly ameliorated by OEFNDC5 (Figure 3B). Based on these, up-regulation of the apoptosis protein Bax and down-regulations of Bcl-2 were all partly abrogated by OE-FNDC5 (Figure 3C).

\section{FNDC5 overexpression suppressed autophagy in BMSCs}

TEM was performed to investigates the effects of OEFNDC5 on autophagy in BMSCs. The representative images indicated that less autophagosomes and autolysosomes were detected in BMSCs-OE-FNDC5 after H/SD induction (Figure $4 A$ ). In addition, immunofluorescence analysis showed that the increased level of LC3 in hypoxia BMSCs was significantly reversed by FNDC5 overexpression (Figure 4B). 
A

C

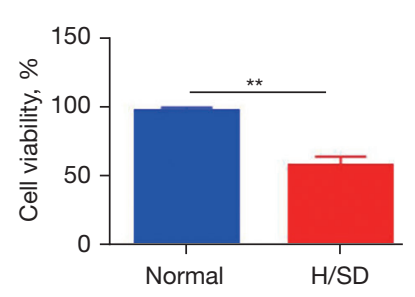

B

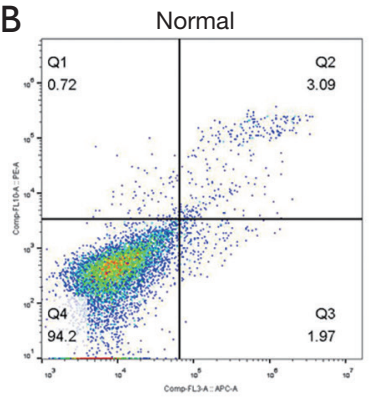

LC3
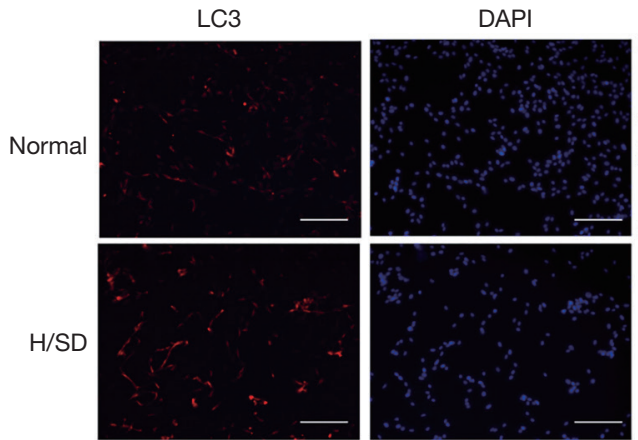
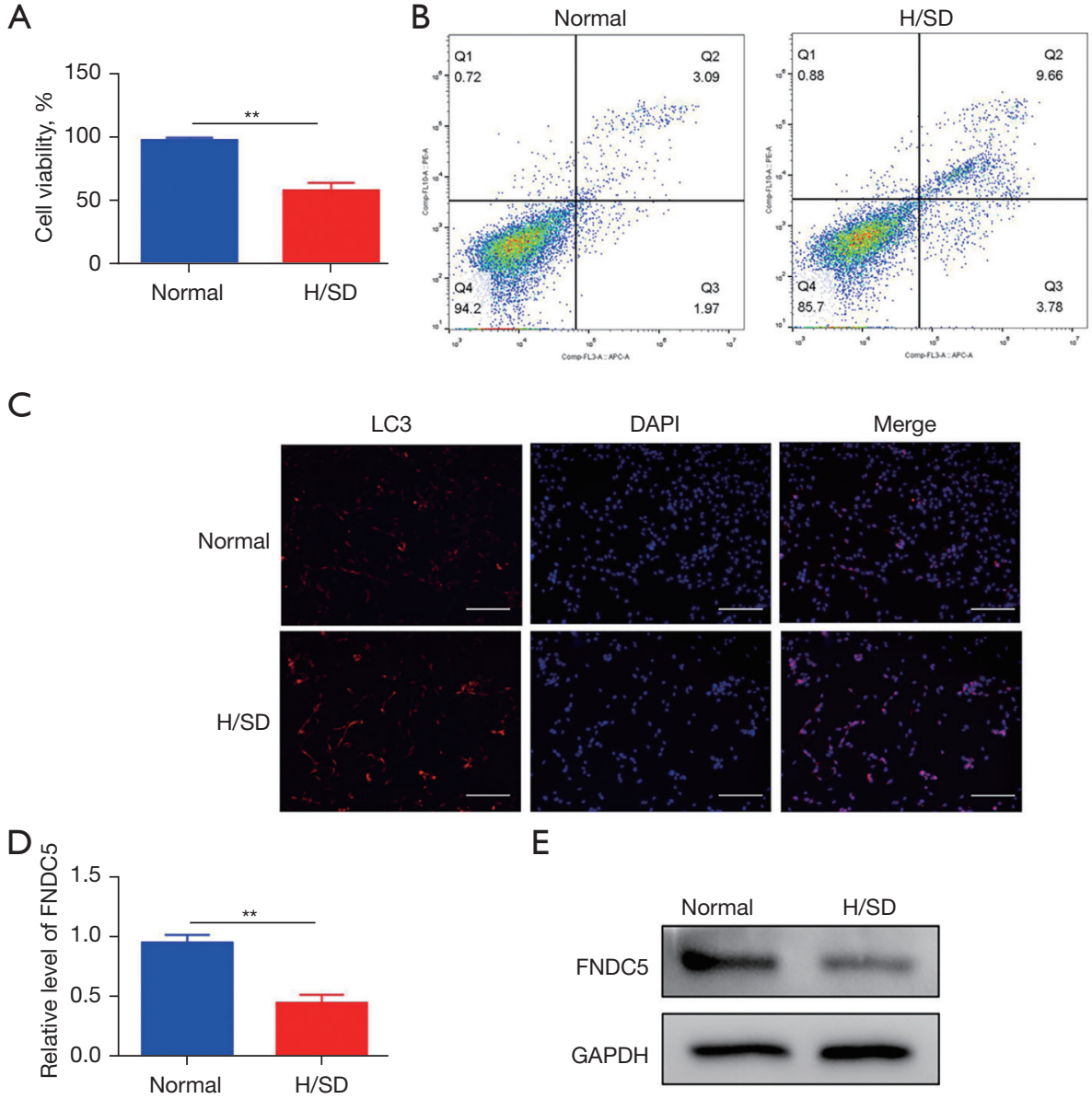

$\mathrm{E}$

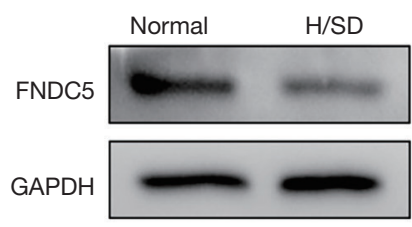

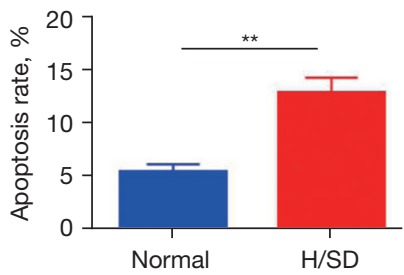
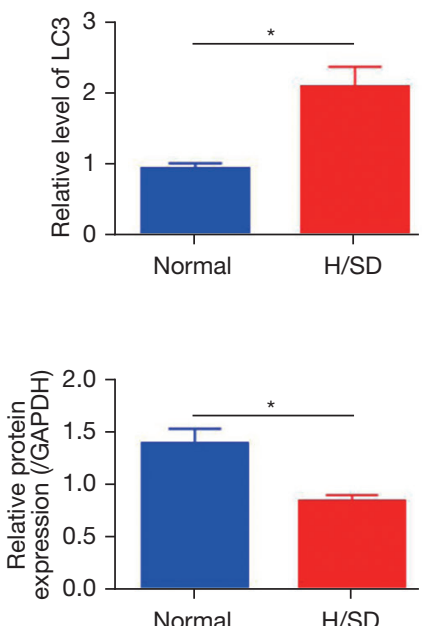

Figure $1 \mathrm{H} / \mathrm{SD}$ exposure decreased cell viability, increased apoptosis and autophagy, and down-regulated the expression of FNDC5 in BMSCs. (A) CCK8 assay indicated the effects of hypoxia (24 h) on the viability of BMSCs. (B) Flow cytometry analysis in BMSCs under hypoxia for $24 \mathrm{~h}$. (C) Representative immunofluorescence images illustrated the levels of LC3 within BMSCs under normal and hypoxia conditions for 24 h. Representative qRT-PCR (D) and western blot results (E) of FNDC5 expression in BMSCs under H/SD. Nuclei were counterstained with DAPI. *, $\mathrm{P}<0.05$; ${ }^{* *}, \mathrm{P}<0.01$. Scale bar $=100 \mu \mathrm{m}$. H/SD, hypoxia/serum deprivation; BMSCs, bone marrow mesenchymal stem cells; LC3, microtubule-associated proteins light chain 3; qRT-PCR, quantitative real-time polymerase chain reaction; DAPI, 4,6-diamidino-2-phenylindole.

Western blot analysis showed that the protein level of autophagy marker, Beclin1, was significantly increased by $\mathrm{H} /$ SD; however, this enhancement was abolished by FNDC5 overexpression. Conversely, p62, which is integrated to LC3 and also serves as a substitutable indicator of autophagy, was down-regulated by H/SD, and this down-regulation was interdicted by OE-FNDC5 (Figure 4C). Together, these results indicated an inhibition role of FNDC5 in BMSCs autophagy.

\section{FNDC5 enbanced the survival rate of engrafted BMSCs}

To track the surviving and proliferative BMSCs, we marked the engrafted BMSCs with EdU. Representative immunofluorescence images showed that most of the BMSCs died after engraftment. In contrast, the proliferative rate of BMSCs-OE-FNDC5 at the edge of the infarct area was significantly higher than those in the BMSCs-OE-NC group. Correspondingly, the RFP-positive cells were more 
A

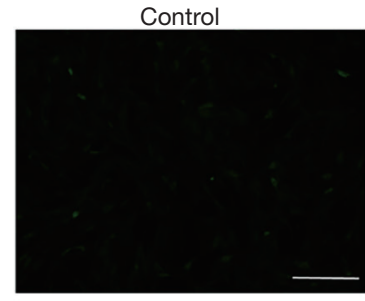

\section{B}

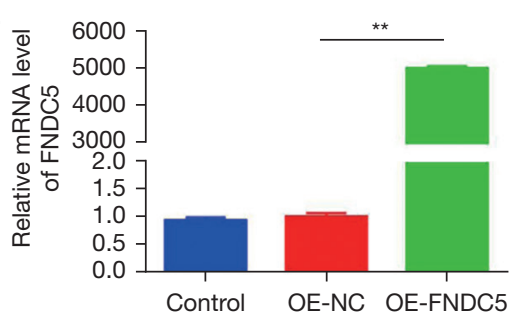

OE-NC

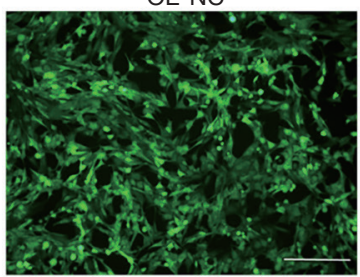

OE-FNDC5

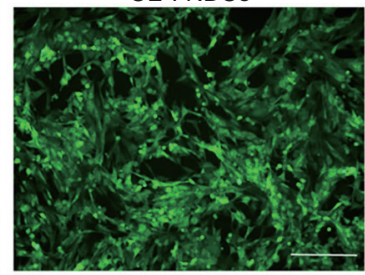

C

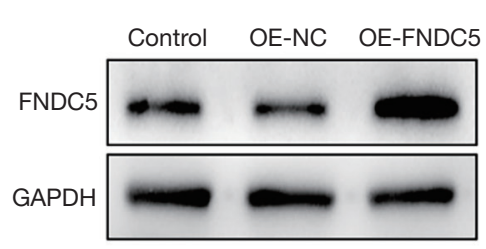

Figure 2 The identification and efficiency of FNDC5 gene transduction. (A) Immunofluorescence staining confirmed the successful overexpression of FNDC5 in BMSCs, and BMSCs-OE-FNDC5 had persistent stability in in vitro cultures. (B) qRT-PCR results exhibited the increased messenger RNA level of FNDC5 in BMSCs transfected by the constructed lentivirus. (C) Western blot images and semiquantification analysis further confirmed the overexpression protein of FNDC5. ** $\mathrm{P}<0.01$. Scale bar $=100 \mu \mathrm{m}$. BMSCs-OE-FNDC5, FNDC5-overexpressing BMSCs.

frequently detected in CI rats administered with BMSCsOE-FNDC5 (Figure 5). These results revealed that OEFNDC5 elevated the survival rate of transplanted BMSCs in the CI edge area.

\section{BMSCs-OE-FNDC5 treatment was more effective in reducing brain infarct volume and alleviating neuronal apoptosis in MCAO rats}

The cerebral infarct volume was measured at 7 days posttransplantation. The TTC staining results showed that BMSCs-OE-FNDC5 engraftment significantly reduced the infarct volume (Figure 6A). The TUNEL assay was performed to detect the neuronal apoptosis at the infarct border, and the staining results (Figure 6B) showed that compared to BMSCs-OE-NC transplantation, BMSCs-OEFNDC5 transplantation obviously decreased the number of TUNEL-positive cells. Overall, these data demonstrated that the therapeutic efficacy of BMSCs transplantation after CI was augmented by FNDC5.

\section{Discussion}

In the present study, we established a CI model in rats using MCAO, and a H/SD-induced hypoxia and ischemia model in BMSCs. Our data indicated that hypoxic stress increased apoptosis, destroyed cell viability, and reduced the protein expression of FNDC5 in BMSCs. OE-FNDC5 ameliorated BMSC injury by suppressing the destructive autophagy induced by continuous hypoxia exposure. Furthermore, OE-FNDC5 significantly improved the survival rate of transplanted BMSCs in the MCAO rat model, thereby markedly reducing the brain infarct volume and alleviating neuronal apoptosis. Taken together, our results provide a novel idea and a scientific basis for modified BMSC strategies in CI therapy.

MSC-based therapy is currently considered to be a promising treatment strategy for CI $(1,2)$. Multiple studies have suggested that MSCs could be beneficial in promoting the regeneration and repair of ischemic cerebral tissue and improve the neurobehavioral function $(3,6,24,25)$. Unfortunately, the survival rate of transplanted MSCs in an ischemic microenvironment remains extremely low $(7,8)$, which seriously impairs the outcome of cell-therapy for CI.

FNDC5 has been recognized as a type I glycosylated transmembrane protein. The extracellular domain of FNDC5, irisin, can be cleaved and released into the bloodstream as a polypeptide (26). Previous research has shown multiple beneficial physiological effects of FNDC5/ irisin, such as regulating energy metabolism, alleviating oxidative stress, neuroprotective effects, and antiinflammatory properties $(27,28)$. Recently, the potential 

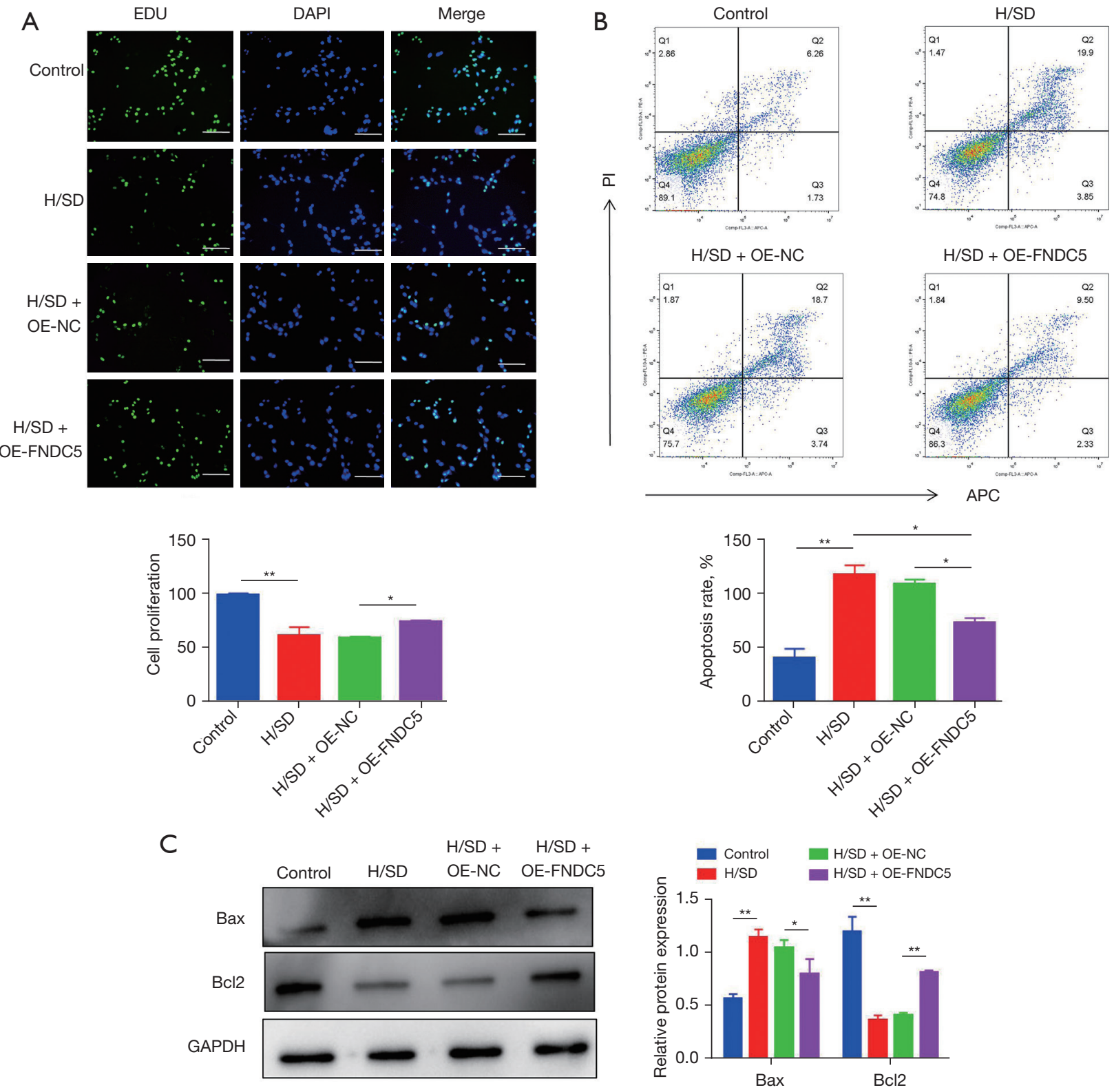

Figure 3 The anti-apoptotic and proliferation promoting effect of OE-FNDC5 on BMSCs subjected to H/SD injury. (A) EdU staining analysis demonstrated that OE-FNDC5 promoted the proliferation of BMSCs subjected to H/SD for 24 h. Proliferation rate $=($ EdU-positive nuclei/DAPI-positive nuclei) $\times 100 \%$. (B) Representative flow cytometric images indicated that OE-FNDC5 decreased the apoptosis rate of BMSCs caused by hypoxia. (C) Western blot results of apoptosis proteins, Bax and Bcl2, in BMSCs with or without OE-FNDC5. * $\mathrm{P}<0.05$; **, $\mathrm{P}<0.01$. Scale bar $=100 \mu \mathrm{m}$. H/SD, hypoxia/serum deprivation; OE-FNDC5, FNDC5-overexpression; EdU, 5-ethynyl-2'-deoxyuridine.

role of FNDC5/irisin in cardiovascular abnormalities, such as atherosclerosis, myocardial infarction, and ischemia stroke, has attracted increasing attention. Researchers believe that high irisin serum levels are highly correlated with cardiovascular disease (CVD), which increases the risk of arteriosclerosis and becomes an index to predict
CI $(10,11,29)$. Exogenous injection of synthetic irisin has been shown to reduce the volume of CI and play a neuroprotective role in a rat MCAO model (9). BMSCs modified by FNDC5 have a higher transplantation survival rate in a hypoxic microenvironment, which decreases the apoptosis of cardiomyocytes and improves cardiac 


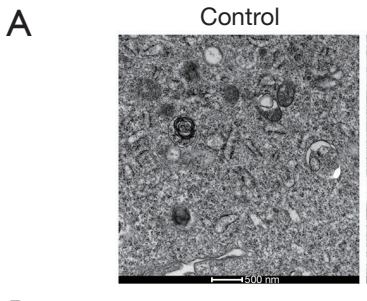

B
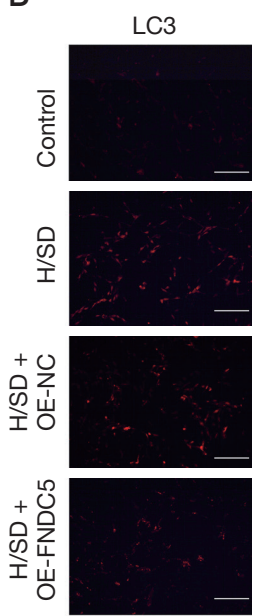

\begin{abstract}
DAPI
\end{abstract}
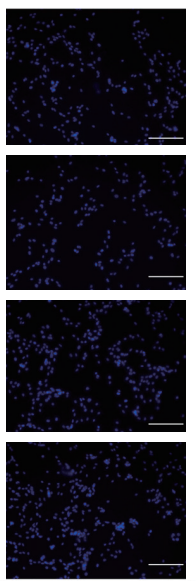

$\mathrm{H} / \mathrm{SD}$

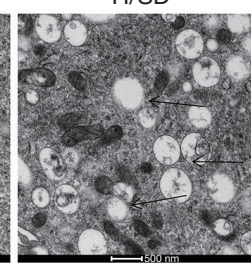

Merge
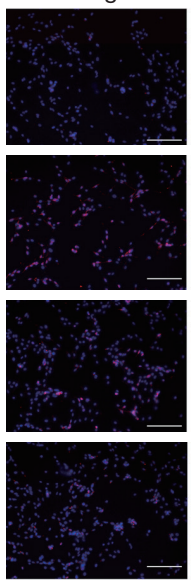

$\mathrm{H} / \mathrm{SD}+\mathrm{OE}-\mathrm{NC}$

$\mathrm{H} / \mathrm{SD}+\mathrm{OE}-\mathrm{FNDC5}$

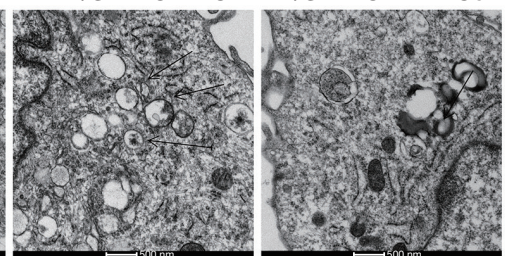

C
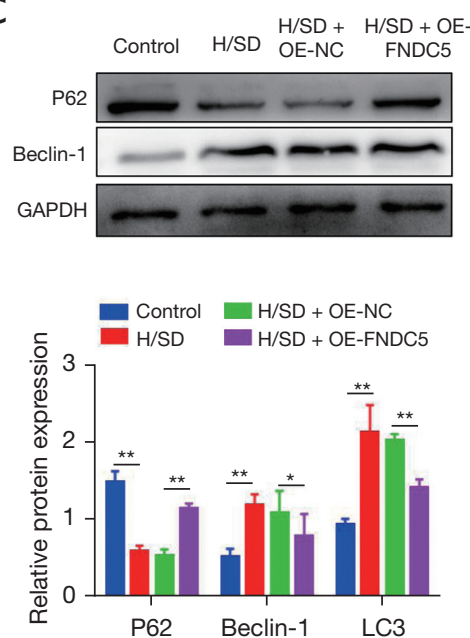

Figure 4 The anti-autophagy effect of FNDC5 in BMSCs subjected to H/SD injury. (A) Transmission electron microscopy images showed the autophagosomes and autolysosomes in BMSCs. (B) Representative immunofluorescence images illustrated the levels of LC3 in BMSCs with or without OE-FNDC5 under hypoxia conditions. (C) Western blot results showed the effect of OE-FNDC5 on the expression of autophagy-related protein, p62, and Beclin-1 in BMSCs. *, $\mathrm{P}<0.05$; ${ }^{*}, \mathrm{P}<0.01$. Scale bar $=100 \mu \mathrm{m}$. H/SD, hypoxia/serum deprivation; OEFNDC5, FNDC5-overexpression.
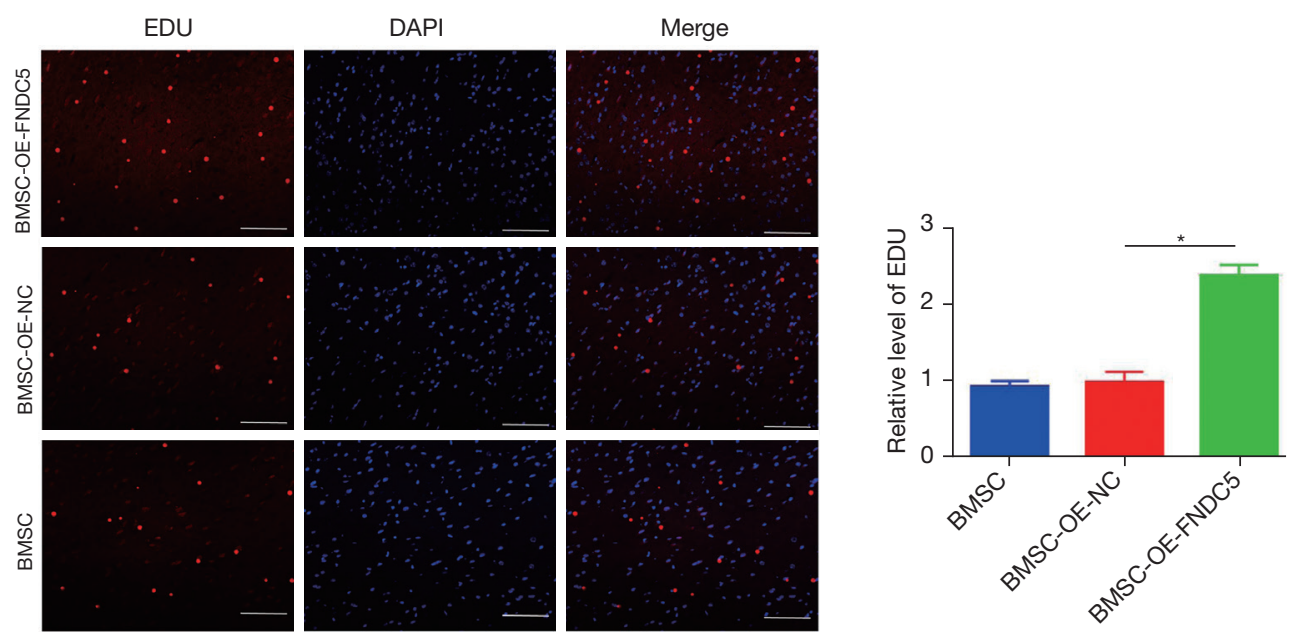

Figure 5 OE-FNDC5 promoted BMSCs survival in the CI border area after transplantation. Representative EdU staining images and semi-quantification of surviving BMSCs-OE-FNDC5, BMSCs-OE-NC, and BMSCs control in the CI edge at 7 days after transplantation. Proliferative cells were indicated by red immunofluorescence of EdU. *, $\mathrm{P}<0.05$. Scale bar $=100 \mu \mathrm{m}$. OE-FNDC5, FNDC5-overexpression; CI, cerebral infarction; BMSCs-OE-FNDC5, FNDC5-overexpressing BMSCs; BMSCs-OE-NC, BMSCs- overexpression-negative control; EdU, 5-ethynyl-2'-deoxyuridine. 

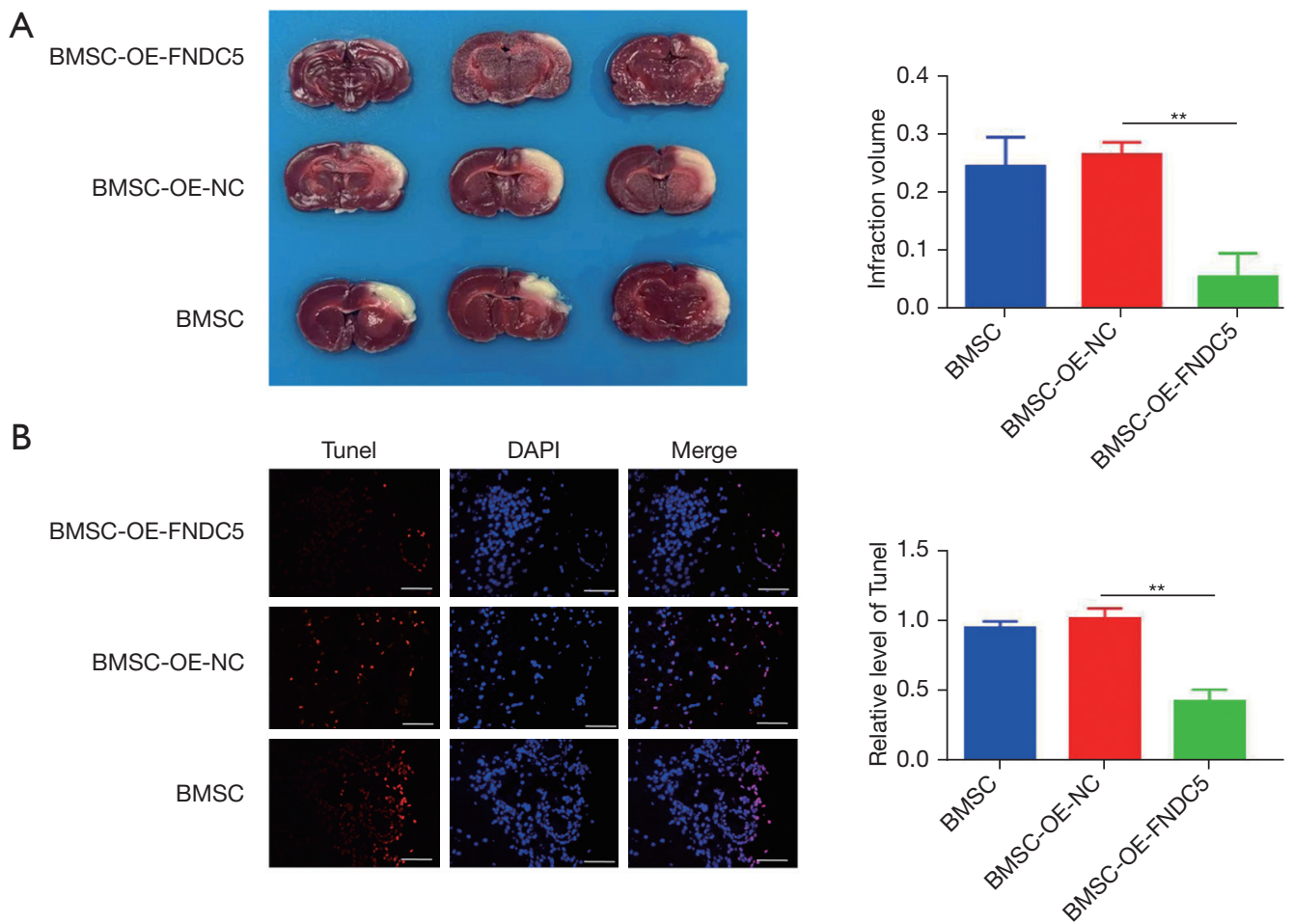

Figure 6 Transplantation of BMSCs-OE-FNDC5 reduced the CI volume of MCAO rats and decreased neuronal apoptosis. (A) The CI volume in rats was evaluated by TTC staining. (B) Immunofluorescence images exhibited that TUNEL-positive neurons were less frequently observed in the MCAO + BMSCs-OE-FNDC5 group compared with the MCAO + BMSCs-OE-NC group. **, P<0.01. Scale bar $=100 \mu \mathrm{m}$. BMSCs-OE-FNDC5, FNDC5-overexpressing BMSCs; BMSCs-OE-NC, BMSCs-overexpression-negative control; CI, cerebral infarction; MCAO, middle cerebral artery occlusion; TTC, 2,3,5-triphenyl tetrazolium chloride; TUNEL, Terminal-deoxynucleoitidyl Transferase Mediated Nick End Labeling.

function (14). In the present study, we also demonstrated that FNDC5 overexpression enhanced the survival rate of BMSCs in hypoxia stress both in vitro and in vivo. In addition, BMSCs-OE-FNDC5 treatment was more effective in reducing the brain infarct volume and alleviating neuronal apoptosis in MCAO rats.

Previous research has demonstrated that the low survival rate of engrafted BMSCs is caused by numerous cell apoptosis and destructive autophagy in a hypoxic environment (8). Growing preclinical evidence suggests that autophagy is a double-edged sword in cardiovascular diseases; it can preserve cell viability under conditions of mild ischemic stress by degrading damaged cellular organelles (30,31), but under conditions of severe ischemia, it can promote cell death (32). The role of FNDC5/irisin on autophagy has been studied only recently, particularly in the last 3 years. Song et al. reported that irisin can improve insulin resistance, possibly in part by inhibiting autophagy via activation of the Phosphatidylinositol 3-kinase/Protein Kinase B (PI3K/Akt) pathway in H9c2 cells (18). In the present study, FNDC5 overexpression inhibited the formation of autophagosomes in BMSCs under hypoxic stress, and reduced the expression of autophagy marker proteins, LC3 and Beclin-1. However, other researchers also found that irisin increases autophagy activity and autophagy flux, and inhibits the expression of the apoptosis signaling pathway in cardiomyocytes (17). The interpretation of these contradictory conclusions could be attributed to the complexity of the autophagy process, which is tightly controlled by a series of factors, including variable pathological environment, diverse protein receptors, and different activation degree of signal molecules.

Although the mechanisms that regulate autophagy are complex and have not been fully elucidated, our study clearly shows that autophagy inhibition caused by OEFNDC5 plays a critical role in BMSC-based therapy for 
treating CI. Our next step is to study the precise signaling transduction mechanism of FNDC5 on the autophagic process in BMSCs. The use of autophagy-related genes (ATGs) knockout might shed light onto its pathophysiology.

\section{Conclusions}

Taken together, the findings presented in this report suggest that FNDC5 overexpression ameliorated hypoxic injury of BMSCs, which could be partly attributed to the inhibition of ischemia-induced destructive autophagy. We also demonstrated that FNDC5 overexpression promoted the survival of BMSCs after engraftment and reduced neuronal apoptosis after CI. Future studies should be aimed at elucidating the molecular mechanisms of FNDC5 in enhancing the therapeutic function of BMSCs.

\section{Acknowledgments}

Funding: This study was supported by grants from the National Natural Science Foundation of China (81671153); the Guangdong Provincial Key Laboratory of Diagnosis and Treatment of Major Neurological Diseases (2020B1212060017); the Guangdong Provincial Clinical Research Center for Neurological Diseases (2020B1111170002); the Southern China International Cooperation Base for Early Intervention and Functional Rehabilitation of Neurological Diseases (2015B050501003, 2020A0505020004); the Guangdong Science and Technology Planning Project (2017B01010015); the Natural Science Foundation of Guangdong Province (2016A030313203); the Guangdong Provincial Engineering Center For Major Neurological Disease Treatment, the Guangdong Provincial Translational Medicine Innovation Platform for Diagnosis and Treatment of Major Neurological Disease, the Science and Technology Project of Kunming Medical and Health Commission (Thousand Project) (No. 2019-03-07-002, No. 2019-03-07-003, No. 2020-03-07-111); the Applied Basic Research of Yunnan Science and Technology Department Associated with Kunming Medical University (No. 202001AY070001260); and the Training plan for Medical Reserve Talents of Yunnan Medical and Health Commission (No. H-2019041).

\section{Footnote}

Reporting Checklist: The authors have completed the ARRIVE reporting checklist. Available at https://atm. amegroups.com/article/view/10.21037/atm-21-6868/rc

Data Sharing Statement: Available at https://atm.amegroups. com/article/view/10.21037/atm-21-6868/dss

Conflicts of Interest: All authors have completed the ICMJE uniform disclosure form (available at https://atm. amegroups.com/article/view/10.21037/atm-21-6868/ coif). HW reports support for the present manuscript from the National Natural Science Foundation of China (81671153), the Guangdong Provincial Key Laboratory of Diagnosis and Treatment of Major Neurological Diseases (2020B1212060017), Guangdong Provincial Clinical Research Center for Neurological Diseases (2020B1111170002), the Southern China International Cooperation Base for Early Intervention and Functional Rehabilitation of Neurological Diseases (2015B050501003, 2020A0505020004), the Science and Technology Project of Kunming Medical and Health Commission (Thousand Project) (No. 2019-03-07-002), and the Training plan for Medical Reserve Talents of Yunnan Medical and Health Commission (No. H-2019041). KL and LL report support for the present manuscript from the National Natural Science Foundation of China (81671153), the Guangdong Provincial Key Laboratory of Diagnosis and Treatment of Major Neurological Diseases (2020B1212060017), Guangdong Provincial Clinical Research Center for Neurological Diseases (2020B1111170002), the Southern China International Cooperation Base for Early Intervention and Functional Rehabilitation of Neurological Diseases (2015B050501003, 2020A0505020004), the Guangdong Science and Technology Planning Project (2017B01010015), the Natural Science Foundation of Guangdong Province (2016A030313203), the Guangdong Provincial Engineering Center For Major Neurological Disease Treatment, the Guangdong Provincial Translational Medicine Innovation Platform for Diagnosis and Treatment of Major Neurological Disease. TW reports supports for the present manuscript from the Science and Technology Project of Kunming Medical and Health Commission (Thousand Project) (No. 2019-03-07-003) and the Applied Basic Research of Yunnan Science and Technology Department Associated with Kunming Medical University (No. 202001AY070001-260). YL reports support for the present manuscript from the Science and Technology Project of Kunming Medical and Health Commission (Thousand Project) (No. 2020-03-07-111). SG reports supports for the present manuscript from the Guangdong 
Science and Technology Planning Project (2017B01010015) and the Natural Science Foundation of Guangdong Province (2016A030313203). The authors have no other conflicts of interest to declare.

Ethical Statement: The authors are accountable for all aspects of the work in ensuring that questions related to the accuracy or integrity of any part of the work are appropriately investigated and resolved. Animal experiments were performed under a project license (No. 2021035) granted by the Animal Care and Use Committee of The Affiliated Yan'an Hospital of Kunming Medical University, in compliance with the institutional guidelines for the care and use of animals.

Open Access Statement: This is an Open Access article distributed in accordance with the Creative Commons Attribution-NonCommercial-NoDerivs 4.0 International License (CC BY-NC-ND 4.0), which permits the noncommercial replication and distribution of the article with the strict proviso that no changes or edits are made and the original work is properly cited (including links to both the formal publication through the relevant DOI and the license). See: https://creativecommons.org/licenses/by-nc-nd/4.0/.

\section{References}

1. Bagheri-Mohammadi S. Protective effects of mesenchymal stem cells on ischemic brain injury: therapeutic perspectives of regenerative medicine. Cell Tissue Bank 2021;22:249-62.

2. Chung JW, Chang WH, Bang OY, et al. Efficacy and Safety of Intravenous Mesenchymal Stem Cells for Ischemic Stroke. Neurology 2021;96:e1012-23.

3. Lin YC, Ko TL, Shih YH, et al. Human umbilical mesenchymal stem cells promote recovery after ischemic stroke. Stroke 2011;42:2045-53.

4. He H, Zeng Q, Huang G, et al. Bone marrow mesenchymal stem cell transplantation exerts neuroprotective effects following cerebral ischemia/ reperfusion injury by inhibiting autophagy via the PI3K/ Akt pathway. Brain Res 2019;1707:124-132.

5. Yuan X, Wang X, Chen C, et al. Bone mesenchymal stem cells ameliorate ischemia/reperfusion-induced damage in renal epithelial cells via microRNA-223. Stem Cell Res Ther 2017;8:146.

6. Rajbhandari S, Beppu M, Takagi T, et al. Ischemia-Induced Multipotent Stem Cells Isolated from Stroke Patients
Exhibit Higher Neurogenic Differentiation Potential than Bone Marrow-Derived Mesenchymal Stem Cells. Stem Cells Dev 2020;29:994-1006.

7. Tambara K, Sakakibara Y, Sakaguchi G, et al. Transplanted skeletal myoblasts can fully replace the infarcted myocardium when they survive in the host in large numbers. Circulation 2003;108 Suppl 1:II259-63.

8. Roh JK, Jung KH, Chu K. Adult stem cell transplantation in stroke: its limitations and prospects. Curr Stem Cell Res Ther 2008;3:185-96.

9. Li DJ, Li YH, Yuan HB, et al. The novel exercise-induced hormone irisin protects against neuronal injury via activation of the Akt and ERK1/2 signaling pathways and contributes to the neuroprotection of physical exercise in cerebral ischemia. Metabolism 2017;68:31-42.

10. Sesti G, Andreozzi F, Fiorentino TV, et al. High circulating irisin levels are associated with insulin resistance and vascular atherosclerosis in a cohort of nondiabetic adult subjects. Acta Diabetol 2014;51:705-13.

11. Wu H, Guo P, Jin Z, et al. Serum levels of irisin predict short-term outcomes in ischemic stroke. Cytokine 2019;122:154303.

12. Liu C, Liu AS, Zhong D, et al. Circular RNA AFF4 modulates osteogenic differentiation in BM-MSCs by activating SMAD1/5 pathway through miR-135a-5p/ FNDC5/Irisin axis. Cell Death Dis 2021;12: 631.

13. Dong Y, Zhang S, Tao J, et al. Fibronectin type III domain-containing protein 5 promotes proliferation and differentiation of goat adipose-derived stem cells. Res Vet Sci 2019;125:351-9.

14. Deng J, Zhang N, Wang Y, et al. FNDC5/irisin improves the therapeutic efficacy of bone marrow-derived mesenchymal stem cells for myocardial infarction. Stem Cell Res Ther 2020;11:228.

15. Hu C, Zhao L, Wu D, et al. Modulating autophagy in mesenchymal stem cells effectively protects against hypoxia- or ischemia-induced injury. Stem Cell Res Ther 2019;10:120.

16. Liu, TY; Xiong, XQ; Ren, XS; et al. FNDC5 Alleviates Hepatosteatosis by Restoring AMPK/mTOR-Mediated Autophagy, Fatty Acid Oxidation, and Lipogenesis in Mice. Diabetes 2016;65:3262-75.

17. Li R, Wang X, Wu S, et al. Irisin ameliorates angiotensin II-induced cardiomyocyte apoptosis through autophagy. J Cell Physiol 2019;234:17578-88.

18. Song R, Zhao $\mathrm{X}$, Cao R, et al. Irisin improves insulin resistance by inhibiting autophagy through the PI3K/Akt pathway in H9c2 cells. Gene 2021;769:145209. 
19. Lourenco MV, Frozza RL, de Freitas GB, et al. Exerciselinked FNDC5/irisin rescues synaptic plasticity and memory defects in Alzheimer's models. Nat Med 2019;25:165-75.

20. Pesce M, Ballerini P, Paolucci T, et al. Irisin and Autophagy: First Update. Int J Mol Sci 2020;21:7587.

21. Longa EZ, Weinstein PR, Carlson S, et al. Reversible middle cerebral artery occlusion without craniectomy in rats. Stroke 1989;20:84-91.

22. Soleimani M, Nadri S. A protocol for isolation and culture of mesenchymal stem cells from mouse bone marrow. Nat Protoc 2009;4:102-6.

23. Frieler RA, Meng H, Duan SZ, et al. Myeloid-specific deletion of the mineralocorticoid receptor reduces infarct volume and alters inflammation during cerebral ischemia. Stroke 2011;42:179-85.

24. Chen J, Li Y, Wang L, et al. Therapeutic benefit of intravenous administration of bone marrow stromal cells after cerebral ischemia in rats. Stroke 2001;32:1005-11.

25. Borlongan CV. Concise Review: Stem Cell Therapy for Stroke Patients: Are We There Yet? Stem Cells Transl Med 2019;8:983-8.

26. Boström P, Wu J, Jedrychowski MP, et al. A PGC1- $\alpha-$

Cite this article as: Wei H, Liu K, Wang T, Li Y, Guo S, Li L. FNDC5 overexpression promotes the survival rate of bone marrow mesenchymal stem cells after transplantation in a rat cerebral infarction model. Ann Transl Med 2022;10(2):90. doi: 10.21037/atm-21-6868 dependent myokine that drives brown-fat-like development of white fat and thermogenesis. Nature 2012;481:463-8.

27. Zhou X, Xu M, Bryant JL, et al. Exercise-induced myokine FNDC5/irisin functions in cardiovascular protection and intracerebral retrieval of synaptic plasticity. Cell Biosci 2019;9:32.

28. Rabiee F, Lachinani L, Ghaedi S, et al. New insights into the cellular activities of Fndc5/Irisin and its signaling pathways. Cell Biosci 2020;10:51.

29. Park KH, Zaichenko L, Brinkoetter M, et al. Circulating irisin in relation to insulin resistance and the metabolic syndrome. J Clin Endocrinol Metab 2013;98:4899-907.

30. Levine B, Kroemer G. Autophagy in the pathogenesis of disease. Cell 2008;132:27-42.

31. Mizushima N, Levine B, Cuervo AM, et al. Autophagy fights disease through cellular self-digestion. Nature 2008;451:1069-75.

32. Xiao C, Wang K, Xu Y, et al. Transplanted Mesenchymal Stem Cells Reduce Autophagic Flux in Infarcted Hearts via the Exosomal Transfer of miR-125b. Circ Res 2018;123:564-78.

(English Language Editor: A. Kassem) 